\title{
Inverse modelling of diffusion-reaction processes with image-type measurement data
}

\author{
A. Penenko ${ }^{1,2 *}$, Z. Mukatova ${ }^{1,2}$, S. Nikolaev ${ }^{3}$, U. Zubairova $^{3}$ \\ ${ }^{1}$ Institute of Computational Mathematics and Mathematical Geophysics SB RAS, Novosibirsk, Russia \\ ${ }^{2}$ Novosibirsk State University, Novosibirsk, Russia \\ ${ }^{3}$ Institute of Cytology and Genetics SB RAS, Novosibirsk, Russia \\ *e-mail: a.penenko@yandex.ru
}

Key words: diffusion-reaction model, inverse problem, image analysis, operator equation

Motivation and Aim: Nonlinear diffusion-reaction models can be applied to the study of development [1] and transport [2] processes in biological tissues. The inverse problems for nonlinear diffusion-reaction models with image-type data arise when the unobservable parameters like synthesis rates or model coefficients are of interest and the additional measurement data is obtained in the form of concentration distribution images (e.g. from microscopy or tomography devices). The aim of the work is to develop an efficient highly parallelizable numerical algorithm for the considered inverse problems. Methods and Algorithms: The inverse problem sensitivity operators constructed from the ensemble of the adjoint equations solutions allow us to transform the inverse problem stated as the system of nonlinear ODE or PDE to the family of nonlinear operator equations depending on the given set of orthogonal functions in the space of the measurement results $[2,3]$. For the solution of the operator equations, we consider Newton-Kantorovich type methods based on the truncated SVD. The operator form of the inverse problem can be further exploited for the analysis and comparison of the different inverse problem statements with the help of the spectral methods.

Results: The inverse problem solution algorithm based on the diffusion-reaction model sensitivity operator and ill-posed operator equations solution methods was developed.

Conclusion: The developed algorithm can be used to solve the inverse problems for the diffusion-reaction models with the image-type measurement data.

Acknowledgements: Supported by the RSF project No. 17-71-10184 in the part of the algorithm development and by RFBR project No. 15-29-04875 in the part of the experimental images acquisition.

\section{References}

1. Nikolaev S.V. et al. (2013) Model of structuring the stem cell niche in shoot apical meristem of Arabidopsis thaliana. Doklady Biological Sciences, 452(1):316-319.

2. Penenko A.V. et al. (2016) Numerical Algorithms for Diffusion Coefficient Identification in Problems of Tissue Engineering. Math. Biol. Bioinf. 11(2):426-444.

3. Penenko A. (2018) Consistent numerical schemes for solving nonlinear inverse source problems with the gradient-type algorithms and the Newton-Kantorovich methods. Siberian J. Num. Math. 21(1):83-99. 